\title{
DESCARTES Y EL CONCEPTO DE ONTOLOGIA
}

Felipe Martinez Marzoa

El empezar recordando que no entendemos por ontología todos lo mismo puede tener la apariencia de uла trivialidad, pues se podria alegar que eso ocurre no sólo con "ontologia", sino con casi cualquier palabra con la que se pretenda designar algún proyecto, y que, puesto que ello es en cierta manera inevitable, más valdria dejar a esos términos globalmente caracterizantes sólo la función de una previa y necesariamente vaga orientación y confiar las precisiones al propio ulterior desarrollo. Esta observación es correcta, en efecto, por razones que conciernen a cualquier caracterización previa de un proyecto $y$, por lo tanto, también a cualquier término con el que un proyecto se designe; pero en el caso de "ontología" lay algo más, algo que por de pronto se traduce en que ni siquiera de manera vaga, aproximativa y puramente material podemos suponer que haya una referencia común cuando unos y otros hablamos de "ontologia", ni aun limitando la consideración a discursos que se reconocen mutuamente la seriedad. Ahora bien, seria de nuevo trivializar el considerar esto como una mera situación de equivocidad o de confusión. Por lo mismo, lo que a continuación voy a decir sobre el término "ontologia" no tiene sólo ni en especial la pretensión de afinar en el uso técnico de las palabras, sino que, si llama la atención sobre algo que ocurre con el empleo de la palabra "ontologia", lo hace sólo porque ello resultará ser mucho más que un problema de equivocos e imprecisiones. Por de pronto, si la palabra "ontologia" responde a cierto modelo de formación de palabras, como "biologia", "epistemologia", etc., no es menos cierto que, en el caso de estas otras palabras. el primer elemento de la composición representa, en el "griego" convencional que empleamos para formar neologismos, algo que también podría decirse en una u otra de las lenguas que nosotros mismos hablamos o podriamos liablar, a saber, en los ejemplos citados, la vida y lo viviente, o bien la ciencia; lo de menos es si esto es o no lo que significan en griego antiguo las palabras del neologismo; en todo caso es lo que significan a efectos de formación de neologismos por nosotros, no por los griegos; $y$, en el caso de "biologia" o "epistemologia", eso que en este orden de cosas signilican los primeros términos es algo que también podemos decir quitándole el velo del neologismo; la "vida" o la "ciencia" son conceptos ciertamente problemáticos, pero al menos lingüisticamente el liecho de nombrarlos está en regla. Ilasta aquí, pues, el neologismo es algo relativamente inocente. En cambio, el intento de retirar el neologismo en el primer clemento del compuesto "ontologia", apelando al mismo teclado de equivalencias meramente convencionales ya aludido, nos conduce cuando menos al borde de lo agramatical, de lo sintácticamente imposible, pues se trataría de un elemento que no podemos identilicar (por ejemplo: identilicar de una lengua a otra dentro de aquellas en las que lo hay o constatar que en otras no lo hay) de otro modo que describiendo cierto útil sintáctico, o, dicho de otra manera, se trata de algo para lo cual la grantitica de las lenguas mismas a las que pertenece (por de pronto nuestras propias lenguas naturales) no genera un uso como lexema pleno, uso que, sin embargo, parece que vendria exigido por el hecho de que en el modelo formacional al que pertenece la palabra "ontologia" el primer elemento de la composición debe significar aquello de lo que se trata, el asunto o el tema o la cosa. Como no se purede hablar de todo en cuarenta minutos, substituiré un desarrollo de esta cuestión por una muy concreta ilustración de la misma. Todo el hablar medieval y moderno de "ente", "el ente". "lo ente", "el ser", comoquiera que esto se diga en cada una de las lenguas implicadas y, desde luego, con total inde- 
pendencia de etimologias y similares, arranca del hecho de que en algún momento, para - supuestamente al menos- traducir algo griego, algo que en griego cra totalmente normal y usual en todas las posibilidades sintácticas de la categoria a la que pertenece y en todos los tipos de habla, en latín se inventó una palabra, es decir, se usó una palabra que sencillamente no existia ni siquicra en el sentido de que los mecanismos de formación que se emplearon para constituirla estuviesen disponibles, una palabra, pues, que sólo podia valer por convenio expreso y "ad hoc". Parece como que precisamente los defensores de la traducibilidad a ultranza tendrían que rasgarse las vestiduras, porque eso que se hizo es en todos los aspectos lo más alejado de ила traducción y lo más parecido al reconocimiento expreso de una intraducibilidad. Me estoy refiriendo, naturalmente, a ens, de cuya fabricación en latin procede todo el uso en las lenguas modernas de "ente", "el ente", "lo ente" (e incluso "el ser", por lo que ahora diré). Al lado de lo que acabo de decir de la inexistencia en latín de ese nombre o adjetivo, es sólo un problema más, aunque ciertamente relevante, el que en todo caso un adjetivo latino no tiene posibilidad correspondiente a la que hay en griego de que se lo emplee en el llamado "neutro singular con articulo" para significar algo asi como "el carácter o la condición de ..." y, en cambio, en el llamado "neutro plural con articulo" para el sentido "colectivo" de "lo ..."; la ausencia de tal posibilidad también en las prolongaciones modernas del recurso latino es lo que nos lleva a veces a recurrir no a "lo ente" ni a "el ente", sino a "el ser", en puntos en que en griego habria tó ón, y a decir en cambio "lo ente" en situaciones en que en griego hay fá ónta. Pero lo que en el presente contexto quiero tomar como ilustración no es la mejor o peor elección de uno u otro término dentro del tinglado de "ente", "el ente", "lo ente", "el ser", sino más bien el que todo el tinglado, con sus correspondencias en cualesquiera lenguas modernas, latinas o no, tiene su origen en aquel acto de fuerza del ens latino. Ahora bien, el acto de fuerza y el subsiguiente tinglado tampoco son constitutivos de error de traducción, pues a fin de cuentas se trata del verbo cópula, y eso es lo que es en griego el verbo al que pertenece ont-. Y, sin embargo, mientras que ont- pertenece con plenitud de posibilidades morfológicas y sintácticas a la lengua común y aparece sin reparo alguno en el poeta "naiv" por excelencia, las palabras latinas y modernas del aludido tinglado son términos innatamente técnicos, sólo posibles en virtud de convenio "ad hoc" y en el marco de una jerga, y tales que, si se insistiese (que no se insiste) en tomarlos desde la lengua, serian - me temo- imposibles. Coincide el hecho de que ese tinglado de términos no se justifica en las lenguas en las que lo encontramos con el hecho de que surgió para traducir de otra lengua algo que, en esa otra lengua, sí pentenece a la lengua misma y no a una jerga convencional; $y$ ambas cosas coinciden con el hecho de que eso que traducimos por "el problema del ser" o "la cuestión del ser" (o, si se quicre, "del ente"), en defínitiva

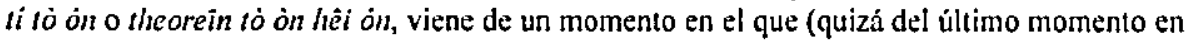
el que, y ello no por casualidad, pero en esto no puedo extenderme ahora) ont- todavia pertenece a la lengua pura y simple. Todas estas coincidencias son una parte de todo lo que nos lleva a sospechar que el carácter innatamente técnico y convencional y a la vez sin embargo la efectiva existencia del complejo terminológico ("ente", "el ente", "Io ente", "el ser") pudiera responder a la vinculación con una problemática cuyo eco por asi decir petrificado se transmite, que de algún modo se conserva en un opaco recuerdo, pero que ya no se entiende. Si es asi, ello habrá de percibirse en que, si en un momento posterior ha llegado a producirse de nuevo autónomamente, originalmente, una problemática que, desde luego en una situación esencialmente distinta, sin embargo conecte en cuanto a la cosa misma con lo que en su momento habia sido ti tò ón y theorein tó òn hêi ón, entonces esa nueva y a la vez antigua problemática ya no habrá podido reconocerse a si misma en las palabras que, sin embargo, se asumian y se asumen como 
traducción de aquellas expresiones griegas, esto es, en los términos del complejo ("ente", "el ente", "lo ente", "el ser"), puesto que el carácter de esos términos está asociado, como hemos visto, a que la problemática ya no se entienda. Precisamente en la medida en que se haya tratado en efecto de un replanteamiento del mismo problema, el título ya no podrá laber sido "el problema del ser". Queda, a lo sumo, abierta la posibilidad de que, en algún momento todavia posterior, el exégeta, es decir, precisamente aquel que ya no puede ser un Aristóteles o Descartes o Leibniz o Kant o Hegel, y que a cambio ha adquirido una nueva capacidad de dejar sonar cada palabra en su propio sonido y, por lo tanto, de barruntar hermenéuticamente la conexión que, en efecto, se cree estar barruntando en lo que por mi parte se está diciendo, queda abierta la posibilidad, digo, de que ese exégeta, al reconocer que cierta problemática - por ejemplo: de Descartes - es de alguna manera el correlato de $t i t o \dot{o}$, y precisamente para exponer de qué manera lo es, exprese esto diciendo que esa problemática es la "ontologia" de - por ejemplo- Descartes o la versión cartesiana del "problema del ser"; comoquiera que de lo dicho se sigue que precisamente eso que en esa clave se llama la "ontologia" no podrá llamarse en el autor interpretado "el problema del ser" ni "del ente" ni, por lo tanto, "ontologia", el exégeta se sentirá apoyado por el hecho de que Descartes mismo precisamente no habla de "el ser" ni de "lo ente" ni de "el ser de lo cnte".

Ciertamente en algunos de los pensadores de la Edad Moderna se liega a conectar internamente la problemática de ellos mismos con el significado de la palabra "ser" en la lengua, pero esto es algo que, a lo sumo, llegará a ocurrir a través del desarrollo de la problemática y que, como corresponde a lo que he dicho, en ningún modo puede constituir el planteamiento de esa problemática, aunque ella sea (o, más bien, precisamente porque es) la que el exégeta debe llamar "ontología". Donde de modo más sistemático y más rico ocurré esa aplicación del significado de la palabra "ser" dentro de la Edad Moderna es en Leibniz, de otra mantra ocurre en Kant, etc.. Por otro lado, ocurre también, concretamente en Hegel, que "ser" designe aquello que precisamente no es todavia (con un "todavia no" intrasistemático) lo que en Hegel constituye el verdadero correlato de tò ón, digo en Hegel, no ciertamente para Hegel mismo, ya que él sí interpreta el ón einai griego en la perspectiva de su "ser". pero esto no hace sino confirmar lo que he diclio de que no es el "ser" hegeliano el verdadero correlato hegeliano del $\dot{o} n$ ếnai griego, pues, al interpretar òn einai en la perspectiva de su "ser", lo que llegel pretende hacer $y$ hace es atar lo griego a un estadio (el de la inmediatez) dentro de su propio sistema. En todo caso, de los dos modos de aparición del recurso linguístico "ser", a saber, la conexión - pero sólo una vez dentro - con el conjunto de la problemática y la restricción a un monento o plano, ninguno tiene relevancia en Descartes, donde sencillamente, en efecto, aquello de lo que se trata no se llana "el ser" o "el ente" o "lo ente". Asi, pues, la interpretación "ontológica", en el sentido hermenéutico que hemos dado a esta expresión, no está aqui oblignda a justificarse ante los problemas que le cree un uso de esos recursos por el pensador mismo interpretado, sino que puede ir directamente al conjunto de su decir y tratar de encontrar en él lo que el exégeta, en la mencionada conexión hermenéutica, se encuentre legitimado para designar como la reasunción o la nueva versión de la cuestión del ser.

l'ara empezar, quien se pregunta cómo, cuándo y bajo quí condiciones estamos legitimados para reconocer algo, para reconocer que algo tiene lugar o que lo hay, está preguntándose qués cabe entender por "tener lugar" o por "haber"; está preguntando por el sentido del "haber" mismo, pues está preguntando qué le reconocemos a aquello de lo que reconocemos que lo hay, qué decimos que "tiene" aquello de lo que decimos que tiene lugar, qué caricter atribuimos a aquello a lo que atribuimos el carácter o la condición de cosa o de ente, o sea, qué es ser. 
Más aún: preguntar qué marcha o qué pasos son necesarios para que acontezca aquella legitimidad del reconocimiento, aquella efectiva presencia de algo, puesto que se trata de la marcha o los pasos necesarios, esto es, inherentes, constitutivos, es ni más ni menos que preguntar qué es lo inherente a tal presencia, qué es, pues, lo constitutivo de la legitimidad de ser reconocido o afirmado, lo constitutivo del tener lugar, esto es, una vez más, qué tiene aquello que tiene lugar, qué es el "lugar" mismo, que es "ser".

La cuestión de cómo puedo llegar a afirmar legítimamente algo, de cómo es posible la legitimidad del reconocimiento, etc., es, pues, ni más ni menos que la cuestión ontológica. la versión ahora encontrable de la pregunta "qué es ser" o "qué hay como constitutivo en el hecho de que algo sea". El que ahora eso se pregunte precisamente de esa manera, a saber, cómo puede llegar a ocurrir que yo esté legitimado para ..., etc., significa, ciertamente, que se lo pregunta de una manera peculiar, distinta de como se lo preguntaron, por ejemplo, los griegos; pero una peculiar manera de preguntarse qué es ser es ni más ni menos que una peculiar manera de hacerse precisamente esa pregunta, es decir, es una peculiar ontologia. Y lo es desde el momento mismo en que de lo que se está hablando es de cómo es posible que se llegue a estar legitimamente seguro etc.; no hace falta esperar a que el pensador emplee fórmulas que suenen a algo asi como "el ser de las cosas en si mismas" o parecido. Si la peculiaridad de esa manera de liacer la pregunta se expresa por de pronto en que el pensador insiste en diversos textos en que está hablando de las cosas en cuanto que tienen que ver con el conocimiento y que, en principio, deja aparte cómo sean "en verdad", este modo de hablar plantea ciertamente un problema interpretativo, pero, justamente para hacerse cargo de él, lo que debe quedar excluido es llamar, por ejemplo, "epistemológico" al orden de cómo se llega a la legitimidad de afirmar y reservar el título de "ontológico" para eso otro, el llamado "ser verdadero", de lo que en esos giros se dice que no se está tratando; pues, muy al contrario, esta reserva de un "ser" más allá o al margen del espacio en el que por el momento se está re-produciendo la problematización del sentido de "ser" significa algo también muy viejo, a saber, que la cuestión ontológica siempre tiene el carácter de una ruptura, del distanciamiento frente a un "ser" cuyo sentido no se problematiza, de manera que con eso de "epistemológico" y "ontológico" se estaria llamando "Io ontológico" precisamente a lo que, en el momento en que se labla, es lo ontológicamente opaco y no problematizado.

He esbozado en qué sentido considero que la cuestión de cómo puede ocurrir que lleguemos a afirmar legitimamente etc. es ni más ni menos que la cuestión ontológica. Ello, si se tiene en cuenta que es esa cuestión de cómo puede llegar a ocurrir que ... la que está en marcha desde la primera linea de las "Meditationes" e incluso desde la primera linea de las "Regulae", comporta que la problemática de Descartes es desde el primer momento y en todo caso "la cuestión del ser"; si la peculiaridad del modo cartesiano de plantear la pregunta comporta los conceptos de método, meditación y duda, lo que esto quiere decir es que en Descartes ya esos conceptos pertenecen a la ontologia misma o son la ontologia misma. Cosa que resulta confirmada por el hecho de que tener el concepto "meditación" y el concepto "duda" es ni más ni menos que ejercer la meditación o la duda y' eso es todo lo que hay, o dicho de otra manera, por el hecho de que exponer en verdad y con detalle qué es la meditación y qué es la duda es ni más ni menos que hacer, como mínimo, todo lo que se hace en las seis "Meditationes" tomadas en su mismo orden.

Con el fin de precisar lo que acabo de decir, me pernitiré esbozar acerca de la marcha de las "Meditaciones" algunas consideraciones que, sin embargo, incluso como esbozo quedarán muy incompletas debido a las limitaciones de extensión de esta intervención. Me permitiré en primer lugar considerar en la "Meditación primera" algo asi como dos etapas; la primera de 
cllas estaria constituida por todo el tramo en el que la duda nos tleva hasta aquel tipo de cosas al que pertenecen las cosas de la aritmética y la geometría, cosas cuya -al menos por el momento- indubitabilidad está constituida por lo que se expresa en la caracteristica consideración de que la certeza de que tres y dos son cinco es exactamente la misma certeza en sueno que en vigilia. Hasta aqui lo que se hace es distinguir entre uno y otro tipo de cosas y reducir el ambito de la certeza a un cierto tipo de ellas; hay, pues, en cierto sentido una reducción material, una reducción de contenido, del ámbito de la certeza. Como sabemos, el progreso de la duda no se detendrá ahi; en todo caso, lo que alıora mismo nos interesa señalar es que el tramo siguiente, el que hemos considerado como segundo tramo de la "Meditación primera", no sólo no liene ya el carácter de reducción de contenido del ámbito de la certeza, sino que en cierto modo suprime o a ciertos efectos niega la reducción material producida en el tramo precedente. Descartes parece querer enfatizar esto al hacer terminar la "Meditación primera" precisamente en el punto en el que, si tomamos el proceso de la duda como reducción de contenido, no que-da nada, lo cual suprime toda apariencia de que pudiera haberse estado produciendo en efecto una reducción del contenido, pues que no quede nada es lo mismo que de nuevo esté todo. Está, en efecto, de nuevo todo; de nuevo hay todo, pero lo que ha cambiado es el sentido del haber, o, para ser más exactos, lo que ha ocurrido es que el sentido del "haber", inicialmente aproblemático, se ha vuelto problema, es decir, se ha puesto en marcha la ontologia. La primera meditación - permitaseme esto como formulación provisional - termina en que no hay nada, lo cual quiere decir que hay todo, pero que el sentido mismo del haber se ha vuelto problemático; ahí queda la cosa, y sólo al día siguiente, reasumiéndose el conjunto de la situación alcanzada, ésta se traduce en que ha quedado el "cogito". Permitaseme un brevisimo inciso. El que sea imprescindible pasar por un momento en el que la desconexión afecta a todo contenido, es decir, no sólo a Jas verdades empíricas, sino también a las del tipo de las matemáticas, es lo mismo que en otro nivel de la exposición aparecerá como una especie de relativización de la necesariedad de las verdades matemáticas 0 , dicho en los términos escolares en los que ha hecho fortuna, como "doctrina de la creación de las verdades eternas"; por obvias razones de liempo, no llegaré en esta intervención hasta ese ulterior nivel, pero he querido mencionar esto para indicar que en el tipo de interpretación que estoy tratando de sugerir esa "doctrina" es central al pensamiento de Descartes y su expresión más cartesiana es precisamente el hecho de que en las "Meditaciones" se tenga que pasar por el momento al que acabo de hacer referencia. Efectuado el inciso, retorno a que la marcha de la meditación se detiene, esto es, cierra etapa, en el momento en que — por asi decir- no hay nada, es decir, en que hay todo, sólo que es el sentido mismo del haber lo que se ha vuelto problemático; esa situación es traducida al dia siguiente en que to que hay es el "cogito". La reducción al "cogito", pues, no es ya una reducción material, óntica, de contenido; no es que antes quedasen las verdades matemáticis y ahora "sólo" quede el "cogito"; por el contrario, el contenido ha recuperado su plenitud inicial, pues al reducirse a nada ha vuelto por ello mismo a ser todo; $y$, en efecto, que hay el "cogito" qujere decir que ónticamente hay todo, pues el contenido del "cogito" es ni más ni menos que todo lo que inicialmente habia; todo, incluso con todas sus diferencias, como la diferencia entre lo necesario y lo contingente, entre lo interno y lo externo, entre lo cierto y lo incierto: el contenido del "cogito" es ni más ni menos que todo eso; en la reducción al "cogito", el cambio no afecta a qué hay, sino a en qué consiste el haber; propiamente ni siquiera se trata de que haya un nuevo "en qué consiste el haber", sino más bien de que ahora hay un "en quí consiste el laber", mientras que antes eso no era problema. Es decir: la reducción al "cogito" significa la problematización no óntica, sino ontológica. Que el "cogito" signitica precisanente esto, lo 
dice a su manera el texto; vamos a ver, aunque sólo sea a título de minima muestra, algo de dónde y cómo el texto dice eso.

La segunda meditación está dedicada a lo que pudiéramos llamar la emergencia y descripción del "cogito". Tras esto, de manera similar a como ocurria con respecto a la primera en el comienzo de la segunda, la tercera comienza por algo que a su vez retoma el conjunto de la segunda; lo hace preguntándose si en esa evidencia del "cogito" o cvidencia de "mi", si en esa evidencia, es decir, apud me, no hay algo de lo que aún no se ha tomado nota expresamente: alia ad quae nondum respexi, es decir: no si hay otras cosas que yo u otras cosas que "cogito", sino si eso mismo, "yo" o "cogito", no tiene ello mismo un significado hasta ahora no explicitamente subrayado; y se contesta que si, que por el hecho de la evidencia "cogito", por el hecho de que sum certus me esse rem cogitantem, a la vez y' por eso mismo scio quid requiratur ut de aliqua re sim cerfus, sé qué se requiere en general para estar legitimamente seguro de algo, esto es: acontece, por el hecho mismo de la evidencia " $y^{\circ} 0$ ", de la evidencia "cogito", el en qué consiste en general que de algo estemos legitimados para la afirmación. es decir, según la sinonimia de la que ya nos hemos ocupado, el en qué consiste en general que algo en efecto sea, que en efecto lo haya. Si ya esto formula con palabras de Descartes lo que hemos dicho de que la remisión al "cogito" significa la problematización del sentido del haber o del sentido de ser, el que esa remisión signifique ni más ni menos que eso se dice de manera más determinada cuando, en la frase inmediatamente siguiente, Descartes justifica to que acaba de decir alegindo que, en efecto, in hac prima cognitione, a saber, en la evidencia "ego cogito", mihil alind est guam ("no hay ni más ni menos que") clara quaedam et disfincta perceptio eius quod affirmo, "la percepción clara y distinta de aquello que afirmo". A una con y constituyendo el mismo acto que la evidencia del "cogito", tiene lugar el en qué consiste en general la legitimidad del reconocimiento, el en qué consiste en general que algo en efecto sea, y ello porque precisamente en la evidencia del "cogito", y constituyendo un mismo acto con ella, tiene lugar el discriminante "percepción clara y distinta". Los adjetivos "claro" y "distinto", en cuya constancia de uso en la obra de Descartes no hace falta insistir, se refieren de manera expresa a la percepción o al conocimiento; ahora bien, por el hecho de que se los emplea sistemáticamente para caracterizar la percepción verdadera como tal, se establece que "cosa" o "ente" o "verdadero" es ni más ni menos que aquello que se percibe cuando la percepción es clara y distinta, con lo cual resulta presupuesto que claridad y distinción son denominaciones que apuntan a lo que hace que la cosa sea en efecto cosa, al ser de lo ente. Ello no sólo es asi por lo que acabamos de leer en Descartes o por lo que sabemos del empleo de esas nociones en su obra, sino que enlaza a la vez con lo hasta aqui dicho sobre la conexión hermenéutica en el empleo de las palabras "ontología" y "cuestión del ser". I lemos vinculado la legitimidad del uso de estas palabras a ecos de significados, y en general de posibilidades de lengua, que funcionaban todavia en Aristóteles y que luego dejaron de funcionar; ont- suena a ousia, a eidos, a phisis; la cuestión no es cómo llegan a Descartes materialmente estas palabras o sus convencionales substitutos latinos, pues es claro que con ellas ocurrira a este respecto lo que ya dijimos que ocurre con "ser"; llegan como representantes del "ser" consabido, de lo ontológicamente opaco, por lo tanto precisamente no como expresión de la cuestión ontológica; independientemente de esto podemos, sin embargo, preguntarnos qué es lo que pese a todo ha quedado del significado de aquellas palabras, por ejemplo: quí es lo que ha quedado del significado de eidos; y lo que ha quedado es esto: presencia y determinación; ciertamente la palabra griega componaba mucho más que eso, y con sólo eso no entenderiamos a Aristóteles ni a Platón; pero lo que ha quedado al perderse la plenitud de significado de la palabra griega ha sido eso: presencia o patencia y' determina- 
ción o de-limitación o de-finición; las palabras cartesianas son respectivamente "claridad" y "distinción", y son en efecto esas las palabras con las que Descartes, al describir con ellas lo que caracteriza como verdadera una percepción, esto es, lo que hace de una percepción presencia de la cosa, describe en definitiva ni más ni menos que la prsencia de la cosa, el que lraya cosa, el que ente sea, el ser de lo ente.

Todo lo que he dicho no hace sino plantear una tarea que sería a la vez la piedra de toque de la via de interpretación sugerida, a saber, interpretar todo lo que resta de las "Meditaciones" como el intento de cumplir con los compromisos que genera el haber asumido la cuestión del ser en el modo en que estamos pretendiendo que la asume Descartes, compromisos que incluy'en el que ese "ser" en definitiva haya de poder también con lo que hasta aqui aparece como el ser consabido, opaco y no problematizado, que "pueda con" él, ya sea en el sentido de integrarlo, ya en el de disolverlo, ya en el de satisfacerlo, ya en cualquier otro (la interpretación diría en cuál). Si ese tipo de interpretación de las "Meditaciones" se consigue, no sólo se habrá conseguido una interpretación de Descartes, sino que también se habrá anudado otro lazo histórico-filosófico. Pues es sabido que lo que llamamos la "metafisica" no toma su sentido de una por asi decir independiente pretensión de acceso a una esfera primera de lo ente, sino de que, incluso como tal pretensión, ella es una cierta expresión (está por ver si inevitable, en todo caso una expresión) de la cuestión del ser. Si el modelo de interpretación de las "Meditaciones" que he sugerido funciona, esa situación en cuanto a la relación entre metalisica y ontologia, situación que es la original, la de Aristóteles, se estaria repitiendo originalmente en Descartes, pues, en efecto, la metalisica de Descartes no seria otra cosa que su ontologia. 